\title{
The Knowledge Production Model of the New Sciences The Case of Translational Medicine
}

\author{
Lauto, Giancarlo; Valentin, Finn
}

Document Version

Accepted author manuscript

Published in:

Technological Forecasting and Social Change

DOI:

10.1016/j.techfore.2016.05.018

Publication date:

2016

\section{License \\ CC BY-NC-ND}

Citation for published version (APA):

Lauto, G., \& Valentin, F. (2016). The Knowledge Production Model of the New Sciences: The Case of Translational Medicine. Technological Forecasting and Social Change, 111, 12-21.

https://doi.org/10.1016/j.techfore.2016.05.018

Link to publication in CBS Research Portal

\section{General rights}

Copyright and moral rights for the publications made accessible in the public portal are retained by the authors and/or other copyright owners and it is a condition of accessing publications that users recognise and abide by the legal requirements associated with these rights.

Take down policy

If you believe that this document breaches copyright please contact us (research.lib@cbs.dk) providing details, and we will remove access to the work immediately and investigate your claim. 


\section{The Knowledge Production Model of the New Sciences: The Case of Translational Medicine \\ Giancarlo Lauto and Finn Valentin}

Journal article (Accepted version)

CITE: The Knowledge Production Model of the New Sciences : The Case of Translational Medicine. / Lauto, Giancarlo; Valentin, Finn. In: Technological

Forecasting and Social Change, Vol. 111, 10.2016, p. 12-21.

DOI: 10.1016/j.techfore.2016.05.018

Uploaded to Research@CBS: July २०17

(C) 2017. This manuscript version is made available under the CC-BY-NC-ND 4.0 license

http://creativecommons.org/licenses/by-nc-nd/4.0/ 
The knowledge production model of the New Sciences: the case of Translational Medicine

\section{Giancarlo Lauto}

Department of Economics and Statistics, University of Udine.

Via Tomadini 30/A, I-33100 Udine, Italy

Research Center on Biotech Business - INO, Copenhagen Business School.

Kilevej 14A, 3, DK-2000 Frederiksberg, Denmark

e-mail: giancarlo.lauto@uniud.it

telephone: +39.0432 .249593$

fax: +39.0432 .249229$

\section{Finn Valentin}

Corresponding author

Research Center on Biotech Business - INO, Copenhagen Business School.

Kilevej 14A, 3, DK-2000 Frederiksberg, Denmark

e-mail: fv.ino@cbs.dk

telephone: +45.3815 .2551$

fax: +45.3815 .2540$

PREPRINT of the article published on

Technological Forecasting and Social Change

http://dx.doi.org/10.1016/j.techfore.2016.05.018 


\begin{abstract}
The tremendous achievements of life sciences research in the last 40 years have brought relatively little improvements to medical practice, suggesting a deficiency of the medical innovation system in capitalizing on these fundamental advances. We argue that a major cause of the poor innovative performance is the slow adaption of the scientific system to the novel research technologies made available by the progress in the life sciences - rather than resistance of practitioners. We interpret the changes in the organization of medical research through the lenses of the theory of New Sciences, which puts forward that the application of novel research technologies promotes new epistemological and methodological approaches to the investigation of complex phenomena, increasing interdisciplinary intellectual exchanges. In Oncology, Translational Research, that embodies the features of a new science, coexists with the standard model of knowledge production in clinical medicine. Our comparison of the two approaches finds that Translational Research allows investigations across diverse and cognitively distant knowledge bases, thanks to the intensive use of research technologies that emerge from fundamental research. Unlike standard studies, the scientific impact of translational studies benefits from the adoption of an interdisciplinary approach. However, translational studies have an overall lower impact than their counterpart.
\end{abstract}

Keywords: Organization of scientific research. Interdisciplinary research. Research technologies. Science-technology link. Cognitive complementarities

\title{
Highlights
}

- We compare "standard" and translational research in Oncology.

- Translational Medicine presents distinctive features of knowledge production. 
- Only Translational Medicine systematically relies on fundamental research technologies.

- Interdisciplinary investigations drive scientific impact only in Translational Medicine.

- Translational Research is associated to a lower scientific impact. 


\section{Introduction}

An effective governance of medical innovation systems is a critical issue for policymakers in many countries - both in the developed and developing World - given the trends of increasing costs of healthcare provision, ageing population, escalation of technological interdependency, and intensification of science-industry linkages (Faulkner 2009; Nicolini 2010; Ryan 2010; Peine et al. 2015). Indeed, medical innovations are the outcome of the co-evolution of different cognitive and institutional domains, such as fundamental biomedical science, clinical practice and medical technology (Gelijns and Rosenberg 1999; Metcalfe et al. 2005; Morlacchi and Nelson 2011; Nelson et al. 2011; Consoli and Ramlogan 2012; Rafols et al. 2014; Kukk et al. 2015). Some studies have shown how advances in the treatment of specific diseases and the introduction of novel approaches, such as personalized medicine, have been based on novel combinations of medical technologies with clinical practices and fundamental research (Amir-Aslani and Mangematin 2010; Tierney et al. 2013; Coccia and Wang 2014; Faulkner, 2015). However, the great expectations brought by the so-called "biotechnology revolution" are still largely unmet. In the last four decades, basic life sciences have attained unprecedented achievements and have generated an entirely new class of research technologies, i.e. novel tools and instrumentation to be used in the process of knowledge production, generating expectations for disruptive changes in a broad range of sciences and industries; despite these advances, the ability of the medical innovation system to generate new and more effective drugs, devices, diagnostics and therapies has been poor (Henderson et al. 1999; Moran 2007; Hopkins et al. 2007).

One explanation for this disappointing performance rests in the delay of the biopharmaceutical industry in identifying suitable business models and in shaping innovation ecosystems favorable to the exploitation these new technologies (Sabatier et al. 2012; Lehoux et al. 2014; Kukk et al. 2015). We 
argue that an equally important reason why medical innovation has progressed relatively slowly - if compared to the great opportunities disclosed by fundamental advances - is to be found in the "science side" of the system.

The availability of novel and more powerful research technologies and the policy pressures demanding an increase in productivity of the medical innovation system, have brought to the emergence of the new field of Translational Medicine. Advocates of Translational Medicine claim that the medical innovation would benefit from insights brought by an intensification of interdisciplinary linkages, the systematic utilization of clinical insights in basic studies, and the prioritization of the solution of patients' problems - rather than disciplinary priorities - in the life sciences research agenda (Marincola 2007; Sablinski 2014). Translational Medicine attracts great interest both within the scientific community and among policymakers, who highlight it as a key strategy for scientific progress in the medical sciences (Grimes 2011; McLeod et al. 2011; Van der Valk et al. 2011; Douglas et al. 2014).

The aim of this paper is to gain insight in the model of knowledge production of Translational Medicine, compared to the "standard" approach that consists in clinically-driven studies that marginally rely on fundamental research; specifically, we compare the two approaches in terms of reliance on novel research technologies, creation of cognitive linkages across disciplines, and drivers of impact on the advance of medical sciences. We take as our case breast cancer research, where the translational approach is particularly prominent. However, also in this field, the "standard" model of research is widely employed and presents limited intellectual exchanges with the translational community (Cambrosio et al. 2006; Jones et al. 2011). For these reasons, breast cancer research is a case that allows the translational and the standard approaches to be rigorously compared. Our study is designed to capitalize on this methodological opportunity. 
We address these issues taking the theory of the "New Sciences" as conceptual reference. The "new sciences" that emerged during the last decades of the $20^{\text {th }}$ century, such as life-, nano- and computer sciences, exploit new and powerful research technologies that allow scientists to investigate the elementary building blocks of extremely complex phenomena such as the human body and its diseases. Systematic application of these research technologies gives rise to a unique model of knowledge production (Bonaccorsi 2008; 2010). The concept of "new science" is used increasingly to gain insights into the features of interdisciplinary fields (e.g. Jansen et al. 2010; Lepori 2011; Heimeriks 2012; Heimeriks and Leydesdorff 2012; Horlings and Gurney 2013).

We contribute to this debate by offering one of the first empirical tests of the theory based on fine-grained comparison of the research conducted in the same medical field according to the principles of new and established sciences. In this way, we bring to light some of the micro-level mechanisms producing tensions and delays in the acceptance of new scientific paradigms (Lakatos and Musgrave 1970). In particular, our finding that translational studies are systematically penalized in terms of impact on subsequent research compared to standard studies adds insights on why scientific communities are slow in adopting interdisciplinary approaches. We suggest that science-internal hindrances represent a major cause of the scarce efficacy of the medical innovation system.

Furthermore, our study contributes to the more general literature on the economics of innovation and technological change, by giving new light on the effect of technological development on the dynamics of science. Studies on the effects of technology on science highlight that research technologies bridge scientific and industrial communities, underpin the emergence of new professional groups and shape new models of collaboration across existing disciplinary boundaries (De Solla Price 1963; Rosenberg 1992; Stokes 1997; Joerges and Shinn 2001; Shinn 2005; Meyer 2007). This paper relates the use of research 
technologies to the differences in the institutional and epistemological dynamics of science, as it shows that only translational studies make an extensive use and contribute to the development of knowledge in the fundamental fields that generate novel research technologies.

The remainder of this paper is organized as follows: we start by defining the theoretical framework from which we derive our hypotheses. After presenting our research design and data, we test the hypotheses, and then discuss our findings.

\section{How research technologies stimulate scientific change}

\subsection{Research technologies and new sciences}

Scientific instrumentation plays a dual role in the process of knowledge production: first, it provides researchers with the technical tools to carry out investigations by enabling the collection and analysis of empirical data; second, it opens up the possibility of investigating new phenomena, thus paving the way to epistemic change (Rosenberg 1992; Collins 1994). The literature on research technologies emphasizes the contribution to epistemic change brought by research tools that emerge from advances in fundamental research. Research technologies offer sets of methods, techniques, standards, and associated concepts that can find application in various settings across disciplinary boundaries. Furthermore, they offer substantial improvements in computing power, accuracy, and precision of research tools (Shinn 2005). Research technologies have been studied mainly from a sociological perspective which looks at how they are invented, their circulation across geographic and institutional settings (primarily, industry and academia), and their contribution to the reconfiguration of occupational groups across disciplines (Joerges and Shinn 2001). Recently, they have been proposed as a major factor underpinning the emergence of a class of "new sciences" representing a model of knowledge production that is radically 
different from that of the established sciences (Bonaccorsi and Thoma, 2007; Bonaccorsi 2008; 2010; Bonaccorsi and Vargas 2010).

The theory claims that, following an established tradition in the core disciplines of natural sciences, new sciences are predicated on reductionist reasoning, especially in terms of methodological stance; however the application of a reductionist approach to complex phenomena (such as the human body or diseases) leads, differently from established sciences, to the proliferation of theories, each addressing different levels of analysis of the phenomenon (e.g. the gene, the molecule, the organ) - typically corresponding to a scientific field. The new sciences attempt to understand also the linkages between theories and observations developed within each of scientific fields. For this reason, a key feature of new sciences is the establishment of cognitive and institutional complementarities, defined respectively as the integration of knowledge developed in heterogeneous disciplinary areas or in multiple institutional settings, such as academia, industry and medical practice. The creation of systematic linkages among concepts and phenomena at the interface between different levels of analysis is a driver of the emergence of novel areas of research that does not imply a weakening of disciplinary boundaries, but rather an increase in the knowledge flows among disciplines (Bonaccorsi 2008; Bonaccorsi and Vargas 2010).

\subsection{Translational medicine as a new science}

Translational Medicine can be regarded as an example of a new science because it relies on the exploitation of novel research technologies, embraces a reductionist research strategy, and progresses by establishing connections among concepts, methods and insights relative to different disciplines by means of research technologies based on fundamental life sciences and computer sciences (Bonaccorsi 2010). 
Traditionally, the cognitive exchange between clinical medicine and the fundamental life sciences has been limited; in fact, medical practice has advanced through cumulative learning on the functioning of specific organs or diseases, or through the systematization of empirical information on patients, without clear guidance from theoretical principles. One of the reasons for the limited reliance of clinical studies on fundamental theories is recognized in the organization of medicine around tight disciplinary specializations defined by diseases (Nelson et al. 2011; Thagard 1999; Bonaccorsi 2010). This applies also to the case of Oncology, which is organized in sub-disciplines defined by the different types of cancers.

However, the many interrelated factors affecting the objects of analysis of clinical research - the patient, the organism, the organ - impede the identification of causal explanations of phenomena addressed by distinct disciplines. For instance, in Oncology, advances in our understanding of the disease may come from the integration on information on how social or environmental factors affect the mutation of the genes responsible for the occurrence of the disease, that is produced in distinct disciplinary domains molecular biology, biochemistry, genetics, clinics, epidemiology, and social and environmental studies (Bonaccorsi 2010). Moreover, this organization of medical research has made it difficult to exploit the fundamental advances brought by the so-called "Molecular Biology revolution" from the mid-1970s. These advances paved the way for the development of research technologies - such as recombinantDNA, monoclonal antibodies, sequencing, polymerase chain reaction, high throughput screening, combinatorial chemistry (Henderson et al. 1999; Judson 1979; Morange 1998; Coccia 2014); furthermore, they gave rise to the ambition to connect clinical investigation to a theoretical framework.

Alone or in combination with information technologies and data-mining facilities, these technologies offered unprecedented opportunities to understand the biochemical causes of physiological and 
pathological phenomena, allowing laboratory analyses on human samples connected to large clinical datasets (Webb and Pass 2004).

As a consequence of the poor exploitation of technology-enabled cross disciplinary explorations, medical research has demonstrated limited ability to translate fundamental advances into new drugs, diagnostics, and devices (Moran 2007; Hopkins et al. 2007). In response to this dissatisfaction, towards the end of 1990s, science policy actively supported "translational" research programs aiming at creating systematic linkages among the different disciplines investigating diseases.

Empirical studies have demonstrated that during the last decades, biomedical research has relied increasingly on inputs generated outside disciplinary boundaries (Porter and Rafols 2009; Rafols et al. 2014) and on the emergence of an interface between fundamental and clinical disciplines (Boyack 2005; Jones 2011). However, most medical studies are conducted according to the "standard", clinically driven approach.

\subsection{The drivers of scientific impact in the new sciences}

To compare the drivers of scientific impact between "new" and "established" sciences, we draw on the notion in Evolutionary Epistemology that knowledge accumulation is a two-step process consisting of the generation of competing explanations for a research problem, and their subsequent selection based on their fitness to resolve that problem (Bradie 1986; Campbell 1974). In this sense, scientific communities develop selection criteria that filter the relevance of theories, approaches, and contributions, and thanks to the positive feedback from the application of successful discoveries (Callon 1994), control the direction of knowledge accumulation. These criteria are signaled through the reputational system of science (Dasgupta and David 1994; Merton 1957). In this system citations are the key informational 
device, since their signals combine priority for single discoveries with their impact on subsequent research. In this perspective, citations indicate the extent to which a given idea "fits" the criteria of selection set by a community (Gittelman and Kogut 2003). Therefore, by identifying the cognitive characteristics of scientific contributions that receive a higher number of citations, we may infer the criteria that a scientific community considers to be important for the accumulation of knowledge.

We conceptualize the characteristics of a given focal body of research using a simple three-stage sequential model of scientific knowledge production (Figure 1). First, we consider its knowledge base, i.e. the way it draws on previous research as "buildings blocks" for its own contribution, particularly the disciplinary diversity of these inputs. Second, we characterize the focal body of research in terms of the disciplinary scope of its own subject matter. Third, we consider its impact on subsequent research in terms of forward citations.

Figure 1 Stages in knowledge production.

\begin{tabular}{|c|c|c|c|}
\hline $\begin{array}{l}\text { Stages in } \\
\text { knowledge } \\
\text { production }\end{array}$ & 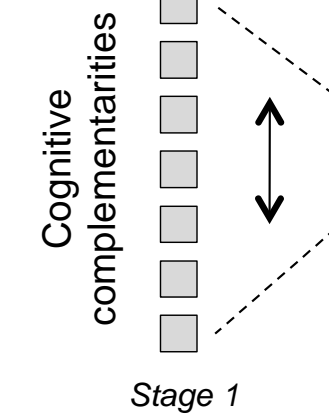 & $\underbrace{}_{\text {Docal field }} \begin{array}{c}\uparrow \\
\text { Discovery } \\
\text { Focal field } \\
\text { Stage 2 }\end{array}$ & $\begin{array}{l}\text { Use of focal } \\
\text { paper in } \\
\text { subsequent } \\
\text { research } \\
\text { (scientific } \\
\text { impact) } \\
\text { Stage 3 }\end{array}$ \\
\hline Key metrics & $\begin{array}{l}\text { Disciplinary } \\
\text { diversity of inputs }\end{array}$ & $\begin{array}{l}\text { Disciplinary areas covered } \\
\text { by focal paper }\end{array}$ & $\begin{array}{l}\text { Citations } \\
\text { received }\end{array}$ \\
\hline
\end{tabular}


Regarding the building blocks for Stage 1, the theory of new sciences highlights the formation of cognitive complementarities. They are expressed in the ability of a focal body of research to link together previously unrelated (cognitively distant) different tools and insights as inputs. A study that ties together previously disconnected inputs offers a search advantage for subsequent research by making it possible for scientists to invoke connections between elements that have been validated in one problem area, to aid the understanding of new findings in other areas (Schilling et al. 2003). According to the theory, establishing such cognitive complementarities is particularly pronounced for new sciences because their reliance on new and powerful research technologies provide them with empirics that can be fruitfully interpreted only by integrating different disciplines. Indeed, principles and standards of research technologies offer scientists operating in distant epistemic communities a shared cognitive platform enabling direct exchanges of theoretical insights, methods, and empirical findings (Shinn 2005). This permits recognition, to a large extent, of analogies among concepts referring to different layers of reality, and the use of methodologies developed in distinct disciplinary traditions. In contrast, standard science relies on lower diversity of research inputs firstly because they are less driven by new research technologies, secondly because they do not receive insights and analogies which migrate across disciplines and domains using new research technologies as their vehicle. Therefore we expect that cognitive complementarities do not drive scientific impact in standard research.

Hypothesis-1: Cognitive complementarities are a driver of scientific impact in new sciences but not in standard science.

The second stage of knowledge production concerns the scope of the subject matter covered by the focal body of research. In standard science the relevance of new contributions is assessed by criteria that are principally internal to each discipline (Whitley 2000), and there will often be reduced receptiveness to 
issues spanning several disciplines (Klein 2008). By contrast, one of the defining characteristics of new sciences is their reach across different and disciplines in their analysis. For example, in Translational Medicine, diseases are explained by reference to factors at the genetic, molecular, or cellular levels. At the same time, these levels have their own specialist disciplines (Bonaccorsi 2010). Hence, new sciences have no intrinsic reservations about research issues transcending single disciplines, nor any systematic preferences.

Hypothesis-2: Increasing scope in the subject matter of a focal contribution detracts from the scientific impact on subsequent research in standard science but not in new sciences.

\section{Research design}

In our empirical analysis of the drivers of scientific impact in breast cancer research we utilize data on publications because they allow us to observe the dynamics of scientific change and relationships among scientific fields reducing the risk of subjective bias (Consoli and Ramlogan 2008). In order to identify the relevant publications, we adopted multiple criteria since previous studies have not discovered any simple bibliometric markers distinguishing translational from "standard" medical research (Luwel and van Wijk 2015). As described further in Appendix-I, our approach is based on the identification of two groups of Lab Leaders, one belonging to the translational and the other to the "standard" epistemic community. For each Lab Leader, we retrieved their articles published in 2003-2007 from the Institute for Scientific Information - Web of Science (ISI-WoS) database. We focused on this period because we want to capture the cognitive dynamics at the early stages of development of this field. We obtained 356 articles - 177 authored by Lab Leaders adopting a translational approach and 179 authored by those 
favoring the standard approach. The low numbers reflect the shortage of cases of Lab Leaders where the classification was unambiguous.

The dependent variable in our study is the impact of an article, based on number of citations received from "original research articles" and review articles up to December 2010. We cleaned the performance measure from self-citations, because, among the scientific community, these are not meaningful for impact (Aksnes and Rip 2009). In order to take into account the time that a paper is available for citation (Years from publication), we adjusted the regression models using the "exposure" option (Long and Freese 2006).

In order to capture the subject Scope of an article, we rely on the categorization of journals into the Subject-Categories used by ISI-WoS. ISI-WoS Subject-Categories indicate the research areas covered by journals; they can be considered proxies for the disciplinary specialization of the articles published in those journals. To examine the scope of a given article, we first associate it with the Subject-Categories describing the journal in which it was published. We then aggregate the Subject-Categories into four Disciplinary Areas (see Appendix-II): Oncology, consisting only of the homonymous Subject-Category, i.e. the core discipline in Breast Cancer Research; Practice-Group, which includes medical specialties defined by their main operational principle; Disease-Fields, which includes medical specialties defined by disease type or organ(-system); RT-Fields, which refers to the fundamental disciplines from which the research technologies applied in medical research originate. We use four dummies to characterize each Disciplinary Area. A fifth dummy, labeled Multiple, takes the value 1 if the focal article is associated with multiple disciplinary areas. This allows us to compare the impact of studies spanning multiple fields (Multiple) with mono-disciplinary studies addressing more fundamental issues (RT-Fields), applied medical research (Oncology, Disease-Fields), and clinical practices and techniques (Practice-Group). 
We capture the role of cognitive complementarities in a focal paper by measuring the diversity of its inputs from previous research. We use the variable Diversity Index which builds on the methodology proposed by Porter et al. (2007) to measure the diversity found in sets of scientific journal publications. The Diversity Index takes value the 0 for papers where all references fall into a single Subject-Category, to a maximum of 1 for i) number of disciplines referenced, ii) their mutual cognitive distance, and iii) the heterogeneity of the distribution of references across Subject-Categories. The last measure is derived from a Matrix of Science based on the frequency of co-citations among Subject-Categories. Subsequently, we generated the categorical variable Diversity with three intervals (low, medium and high), defined at the cut-off points of the $33^{\text {rd }}$ and $67^{\text {th }}$ centiles of the distribution of the Diversity Index.

We distinguish between the standard and the translational Approaches with a dummy that takes the value of 1 for publications produced by translational Labs and 0 for standard science Labs. We appreciate how the approaches differ in terms of cognitive complementarities and subject scope, by including two sets of dummy variables. The first set focuses on the differences between approaches in studies that span multiple disciplinary areas and those that are contained within one disciplinary area. To this purpose, first we create the variable Monosciplinary, that characterizes papers classified exclusively in one of the following disciplinary areas: RT-Fields, Oncology, Disease-Fields and Practice-Group; then, we consider all the possible categories produced by the combination of Approach with Multiple and Monosciplinary: Translational-Multiple, Translational-Monodisciplinary, Standard-Multiple, and Standard-Monodsiciplinary. The second set is based on the six categories defined by combinations of Approach and Diversity, i.e. Translational-Low Diversity; Translational-Medium Diversity; Translational-High Diversity; Standard-Low Diversity; Standard-Medium Diversity; Standard -High Diversity. 
We apply seven controls. Since theoretical breast cancer research studies tend to receive more citations (Lewison et al. 2010), we control for the focal paper's orientation towards more basic or more applied issues. We characterize this orientation by means of the CHI-classification ${ }^{1}$ which distinguishes between: 1) clinical observation, 2) clinical mix, 3) clinical investigation, and 4) basic research (Narin et al. 1976). Following, Van Looy et al. (2006), we aggregate Levels-3 and 4 into a single category representing a basic orientation, and refer to Levels-1 and 2 as clinical orientation. The variable Orientation takes the value 1 for basic orientation and 0 otherwise.

Within a Lab Leader's research program, there may be synergies among individual projects. A later article building on earlier investigations might exploit synergies and cumulativeness; on the other hand, subsequent contributions might be unrelated to the author's previous work. In order to account for these effects, we considered the dummy Cumulative which takes the value 1 if the focal article cites the LabLeader's previous production in the period considered.

We control for the extent to which a paper builds on a novel vs. mature knowledge base. We avoid the distorting effect of citation of "classical" contributions, considering the age of the most recent quartile of its references. The dummy (Age) identifies articles that are based on a mature knowledge base: it takes the value 1 for papers whose age of the most recent quartile of their references is higher than the average for the sample, i.e. three years.

\footnotetext{
${ }^{1}$ We are aware of the limits the CHI-classification for measuring "basicness" (Tijssen 2010), and the limits of Subject-Categories to define disciplines (Pudovkin and Garfield 2002). We base our variables on these tools because of their widespread diffusion in studies of scientists' productivity. The former is used by e.g. Van Looy et al. (2006); the latter by e.g. Ponomariov and Boardman (2010).
} 
Collaborative research is known to have greater impact than individual research (e.g. Adams et al. 2005), so we control for research team size. A set of dummies is used to distinguish among "Small collaborations" with less than 9 co-authors, "Medium-sized collaborations" with 9 to 11 co-authors, and "Large collaborations" with more than 11 authors. The cut-off points used to define the classes are the $33^{\text {rd }}$ and $67^{\text {th }}$ percentile of the distribution of the size of the research team.

We use a dummy to identify each Lab Leader to capture individual factors - e.g. individual talent, organization of the team of inner collaborators, age of the team.

We employ negative binomial regression models since our dependent variable - number of citations received by each article - is a non-negative count with over-dispersed distribution. For ease of interpretation, we display Incidence Rate Ratios (IRR). An IRR greater than 1 indicates a positive contribution to citations, while an IRR between 0 and 1 indicates a negative effect. We checked for heteroskedasticity with graphical and numerical techniques. We used robust standard errors. We can exclude that the results of the regressions are substantially biased by multi-collinearity since the maximum Variance Inflation Factor is below the threshold of 10 in all the models. All models include 356 observations. Significance at $0.10,0.05$ and 0.01 levels is indicated by $*, * *$, and $* * *$ respectively.

\section{Results}

\subsection{Descriptive statistics}

The descriptive statistics presented in Table 1 suggest that translational and standard medical research involve distinct specialization patterns regarding both disciplinary specialization and input diversity. In both approaches, more than half of the production is represented by mono-disciplinary studies in Oncology. Differences emerge outside the core disciplinary domain, and in the way this core is 
addressed: Translational studies often address the areas of RT-Fields and Disease-Fields, which account for a negligible share of standard research. Standard research includes comparatively more investigations of Practice-Group. The share of articles covering multiple disciplinary areas is similar, indicating that standard research also regularly addresses issues referring to different disciplinary domains; however, there is a substantial qualitative difference between the two approaches in this regard. In fact, $54 \%$ of the translational studies characterized by broad scope, combine RT-Fields with other areas, while standard research rarely makes contributions that matter for both RT-Fields and other disciplinary areas. This result underscores the substantial difference between the model of knowledge production in Translational Medicine and standard medical research.

The average of the Diversity Index is $30 \%$ higher for translational than standard research; the majority (43\%) of translational studies show high levels of Diversity, while standard studies show low levels of Diversity (48\%). Translational Medicine shows a clear orientation towards more basic issues, but this is to be expected based on the procedure used to build the sample. As for the other controls, there are no significant differences between the approaches.

\section{Table 1 Descriptive statistics}

\begin{tabular}{|c|c|c|c|c|c|c|c|}
\hline Variable & Total & & $\begin{array}{c}\text { Translation } \\
\text { al }\end{array}$ & & Standard & & Test \\
\hline \multirow[t]{2}{*}{ Total n. of articles } & 356 & & \begin{tabular}{|c|}
177 \\
\end{tabular} & & 179 & & \\
\hline & $\begin{array}{c}\text { Mean } \\
\text { (Std. Dev.) }\end{array}$ & Median & $\begin{array}{c}\text { Mean } \\
\text { (Std. Dev.) }\end{array}$ & Median & $\begin{array}{c}\text { Mean } \\
\text { (Std. Dev.) }\end{array}$ & Median & 1 \\
\hline N. of Citations & $\begin{array}{c}26.736 \\
(55.489) \\
\end{array}$ & 11 & $\begin{array}{c}27.395 \\
(54.443)\end{array}$ & 12 & $\begin{array}{c}26.084 \\
(56.650) \\
\end{array}$ & 11 & $-1.659^{*}$ \\
\hline \multirow[t]{2}{*}{ Diversity Index } & $\begin{array}{c}0.449 \\
(0.151) \\
\end{array}$ & 0.471 & $\begin{array}{c}0.508 \\
(0.110) \\
\end{array}$ & 0.500 & $\begin{array}{c}0.391 \\
(0.164) \\
\end{array}$ & 0.413 & $-6.798^{* * *}$ \\
\hline & n. & $\%$ & n. & $\%$ & n. & $\%$ & 2 \\
\hline \multicolumn{8}{|l|}{ Diversity } \\
\hline Low & 117 & 32.87 & 31 & 17.51 & 86 & 48.04 & $\mathrm{p}=0.000$ \\
\hline Medium & 122 & 34.27 & 70 & 39.54 & 52 & 29.05 & $\mathrm{p}=0.044$ \\
\hline High & 117 & 32.87 & 76 & 42.94 & 41 & 22.90 & $\mathrm{p}=0.000$ \\
\hline \multicolumn{8}{|l|}{ Disciplinary Areas } \\
\hline Oncology & 198 & 55.62 & 94 & 53.11 & 104 & 58.10 & $\mathrm{p}=0.393$ \\
\hline
\end{tabular}




\begin{tabular}{|l|c|c|c|c|c|c|c|}
\hline Disease-Fields & 17 & 4.78 & 12 & 6.78 & 5 & 2.79 & $\mathrm{p}=0.087$ \\
\hline Practice-Group & 45 & 12.64 & 13 & 7.34 & 32 & 17.88 & $\mathrm{p}=0.004$ \\
\hline RT-Fields & 18 & 5.06 & 17 & 9.60 & 1 & 0.56 & $\mathrm{p}=0.000$ \\
\hline Multiple & 78 & 21.91 & 41 & 23.16 & 37 & 20.67 & $\mathrm{p}=0.609$ \\
\hline of which: & & & & & & & \\
\hline RT-Fields + Other & 25 & 32.05 & 22 & 53.66 & 3 & 8.11 & $p=0.000$ \\
\hline Other combinations & 53 & 67.95 & 19 & 46.34 & 34 & 91.89 & $p=0.000$ \\
\hline Orientation & 111 & 31.18 & 90 & 50.85 & 21 & 11.73 & $\mathrm{p}=0.000$ \\
\hline Cumulative & 196 & 55.06 & 117 & 66.10 & 79 & 44.13 & $\mathrm{p}=0.328$ \\
\hline Team-Size & & & & & & & \\
\hline Small & 130 & 36.52 & 62 & 35.03 & 68 & 37.99 & $\mathrm{p}=0.583$ \\
\hline Medium & 95 & 26.69 & 50 & 28.25 & 45 & 25.14 & $\mathrm{p}=0.550$ \\
\hline Large & 131 & 36.80 & 65 & 36.72 & 66 & 36.87 & $\mathrm{p}=0.999$ \\
\hline Age & 123 & 34.55 & 65 & 36.72 & 58 & 32.40 & $\mathrm{p}=0.496$ \\
\hline Publication Year & & & & & & & \\
\hline 2003 & 80 & 22.47 & 38 & 21.47 & 42 & 23.46 & $\mathrm{p}=0.704$ \\
\hline 2004 & 60 & 16.85 & 31 & 17.51 & 29 & 16.20 & $\mathrm{p}=0.778$ \\
\hline 2005 & 70 & 19.66 & 35 & 19.77 & 35 & 19.55 & $\mathrm{p}=0.999$ \\
\hline 2006 & 64 & 17.98 & 31 & 17.51 & 33 & 18.44 & $\mathrm{p}=0.890$ \\
\hline 2007 & 82 & 23.03 & 42 & 23.73 & 40 & 22.35 & $\mathrm{p}=0.802$ \\
\hline
\end{tabular}

${ }^{1}$ Wilcoxon-Mann-Whitney test; ${ }^{2}$ Fisher-test.

We deepen our examination of the Diversity Index by looking at its components, i.e. we analyze the distribution of references cited by focal papers across disciplinary areas. For each focal paper we calculate the share of references to four Disciplinary Areas $^{2}$; we summarize the mean and median values in Table 2 which presents the results for the entire set of articles and the results disaggregated by level of Diversity. This highlights the type of knowledge bases associated with low, medium, and high levels of cognitive diversity.

The knowledge base in standard research is focused heavily on Oncology: in the median article we find $50 \%$ of references draw on this Disciplinary Area. Studies in Oncology offer a major contribution also to translational research, although the approach is significantly less reliant on this kind of input (the

\footnotetext{
${ }^{2}$ If a reference refers to multiple disciplinary areas, we attribute it to each of them. Thus, the category "Multiple" is not considered in this analysis.
} 
median share of references to Oncology is 40\%). There are important differences between the two approaches in relation to the weight of the other disciplinary areas and levels of Diversity.

In the standard approach, an increase in the level of Diversity corresponds to a decrease in the inputs from Oncology: in the case of highly integrative articles, we find that the difference between the two approaches is no longer significant (on average, $32.8 \%$ vs. 29.5\%). What are the other knowledge bases utilized by these two approaches when they pursue cognitive diversity? Table 2 shows that the knowledge base in the standard approach is characterized by a strong presence of inputs from the Practice-Group at each level of Diversity, and that Disease Fields are an important source of knowledge for highly integrative papers. RT-Fields represent marginal knowledge at each level of Diversity.

The knowledge base is substantially different for translational research. RT-Fields are an important source of inputs at each level of diversity, and are the most important source, at the expense of Oncology, for highly integrative work. In contrast to what we observe in standard research, the contribution of Practice-Group declines as research becomes more integrative. The pattern for Disease-Fields inputs is similar for both areas.

Table 2 Mean and median (in parentheses) share of cited references by disciplinary area, disaggregated by level of Diversity of the focal paper.

\begin{tabular}{|l|l|c|c|c|c|}
\hline & & Total & \multicolumn{3}{|c|}{ Diversity } \\
\hline & & & Low & Medium & High \\
\hline Approach & $\begin{array}{l}\text { Disciplinary area of } \\
\text { references }\end{array}$ & & & & \\
\hline Standard & Oncology & $0.512^{* * *}(0.500)$ & $0.640^{* * *}(0.689)$ & $0.445^{*}(0.461)$ & $0.328(0.333)$ \\
\hline & Disease-Fields & $0.115^{* * *}(0.053)$ & $0.062(0.032)$ & $0.161(0.054)$ & $0.236(0.188)$ \\
\hline & RT-Fields & $0.059^{* * *}(0.018)$ & $0.024^{* * *}(0.000)$ & $0.089^{* * *}(0.589)$ & $0.102^{* * *}(0.091)$ \\
\hline & Practice & $0.314^{* * *}(0.276)$ & $0.277(0.208)$ & $0.360^{* * *}(0.350)$ & $0.334^{* * *}(0.313)$ \\
\hline & & & & & \\
\hline Translational & Oncology & $0.392(0.403)$ & $0.552(0.591)$ & $0.427(0.442)$ & $0.295(0.300)$ \\
\hline & Disease-Field & $0.140(0.090)$ & $0.059(0.032)$ & $0.081(0.045)$ & $0.227(0.172)$ \\
\hline & RT-Fields & $0.273(0.259)$ & $0.171(0.100)$ & $0.278(0.260)$ & $0.388(0.315)$ \\
\hline & Practice & $0.195(0.143)$ & $0.212(0.143)$ & $0.213(0.128)$ & $0.169(0.153)$ \\
\hline
\end{tabular}


Note: Asterisks indicate the level of significance of difference between the distributions of the share of references in each disciplinary area of translational and standard studies. Differences are tested with Wilcoxon-Mann-Whitney test.

This evidence indicates that the disciplinary diversity of the inputs to the two approaches is the result of substantially different patterns of knowledge production: Standard research tends to combine knowledge on Oncology or other diseases with practical knowledge produced in the Practice-Group, with little or no contribution from RT-Fields. By contrast, Translational Medicine relies heavily on RT-Fields at each level of diversity. It is useful to bear these different input-profiles in mind when interpreting the Diversity Index. For the standard approach, high diversity refers primarily to rare combinations of building blocks from Oncology and Practice-Group. For the translational approach, high diversity refers to a much greater extent to combinations of RT-Fields with Oncology and Practice-Group. These results provide evidence to the theoretical expectation that a new science will utilize research technologies in order to establish cognitive complementarities.

We next examine the drivers of scientific performance by looking at the average and mean citation level of translational and standard research for the two key cognitive drivers: Diversity Index and Disciplinary Areas (Table 3).

Table 3 Scientific impact across key cognitive dimensions in Translational and Standard research

\begin{tabular}{|l|c|c|c|c|}
\hline & Translational & & Standard & \\
\hline & Mean (Std. Dev.) & Median & Mean (Std. Dev.) & Median \\
\hline Diversity & & & & 12.5 \\
\hline Low & $18.68(17.20)$ & 12 & $24.86(42.00)$ & 9 \\
\hline Medium & $40.57(77.86)$ & 17.5 & $26.13(67.87)$ & 12 \\
\hline High & $18.82(31.50)$ & 8.5 & $28.59(68.19)$ & 13.5 \\
\hline Disciplinary Area & & & & $24.93(38.27)$ \\
\hline Oncology & $24.65(30.21)$ & 11 & $7.60(7.33)$ & 3 \\
\hline Disease-Fields & $13.67(8.52)$ & 13 & \\
\hline
\end{tabular}




\begin{tabular}{|l|c|c|c|c|}
\hline Practice-Group & $77.08(167.03)$ & 12 & $54.53(110.41)$ & 13 \\
\hline RT-Fields & $32.94(54.54)$ & 11 & $4.00(-)$ & 4 \\
\hline Multiple & $19.66(19.75)$ & 14 & $7.81(6.88)$ & 5 \\
\hline
\end{tabular}

The relationship between Diversity Index and level of performance seems to differ between the approaches: In the standard approach we find an increase in the mean number of citations as we move from low to high Diversity, while the median number of citations received by papers with low and high Diversity is respectively similar and higher than for those with medium Diversity. This indicates that, even in the standard approach there are gains deriving from knowledge diversity; However, they are not systematic, but rather are driven by few highly integrative papers that receive a high number of citations. Translational studies, by contrast, show higher citation rates than standard ones only for a medium level of Diversity, at other levels standard studies have a higher impact.

In relation to Disciplinary Areas, we find that the median citation level of translational studies is similar across areas, while there are large differences among means. Again, these differences are due to a few highly cited papers. In the standard approach, the median articles in Oncology and Practice Group show similar performance, which is slightly higher than their translational counterparts, while studies across multiple disciplines have considerably lower mean and median impact.

Overall, these results indicate the strength of standard science in Oncology and Practice Group, while translational studies achieve a substantially similar level of performance in all Disciplinary Areas. The standard approach presents too few observations in RT-Fields and Disease-Fields for meaningful comparison with translational research. 


\subsection{Regression analysis}

In order to validate the descriptive findings using more robust econometric tools, we employ a series of negative binomial regression models to test the hypotheses about the effect of cognitive factors on the scientific impact of discoveries emerging from translational and standard approaches.

Our dependent variable is the number of citations obtained by an article. After presenting the controlsonly Model 1, in Model 2 we introduce the key explanatory variables: Disciplinary Areas, Diversity, and Approach. In Models 3 and 4 we include the categorical variables expressing the joint effect of Approach and Diversity, and Approach and Multiple. These variables allow us to assess whether the two approaches differ significantly for drivers of scientific impact. 
Table 4 Regression models

\begin{tabular}{|c|c|c|c|c|}
\hline & $\begin{array}{c}\text { Model } 1 \\
\text { LPR }(\text { Std Frr })\end{array}$ & $\begin{array}{c}\text { Model } 2 \\
\text { LPP }(\text { Std Frr })\end{array}$ & $\begin{array}{c}\text { Model 3 } \\
\text { JPP (Std Frr) }\end{array}$ & $\begin{array}{c}\text { Model } 4 \\
\text { LP }(\text { Std Frr })\end{array}$ \\
\hline & & IRR (Std. Err.) & IRR (Std. Err.) & \\
\hline $\begin{array}{l}\text { Approach } \\
\text { Baseline: Standard }\end{array}$ & & $0.239^{* * * *}(0.088)$ & & \\
\hline \multicolumn{5}{|l|}{$\begin{array}{l}\text { Diversity } \\
\text { Baseline: Low }\end{array}$} \\
\hline Medium & & $0.897(0.139)$ & & $0.916(0.142)$ \\
\hline High & & $1.002(0.174)$ & & $0.975(0.167)$ \\
\hline \multicolumn{5}{|l|}{$\begin{array}{l}\text { Disciplinary Area } \\
\text { Baseline: Oncology }\end{array}$} \\
\hline Disease Field & & $0.628^{* *}(0.135)$ & $0.632^{* *}(0.138)$ & $0.664^{*}(0.146)$ \\
\hline RT-Fields & & $0.931(0.308)$ & $0.942(0.303)$ & $1.040(0.362)$ \\
\hline Practice Group & & $2.133^{* * * *}(0.532)$ & $2.157^{* * * *}(0.528)$ & $2.019^{* * * *}(0.508)$ \\
\hline Multiple & & $0.679^{* * * *}(0.095)$ & $0.715^{* *}(0.101)$ & \\
\hline \multicolumn{5}{|c|}{$\begin{array}{l}\text { Multiple x Approach } \\
\text { Baseline: Oncology, Standard }\end{array}$} \\
\hline Multiple, Standard & & & & $0.449^{* * * *}(0.084)$ \\
\hline Oncology, Translational & & & & $0.219^{* * *}(0.080)$ \\
\hline Multiple, Translational & & & & $0.201^{* * * *}(0.078)$ \\
\hline \multicolumn{5}{|l|}{$\begin{array}{l}\text { Diversity x Approach } \\
\text { Baseline: Low, Standard }\end{array}$} \\
\hline Medium, Standard & & & $0.603^{* *}(0.129)$ & \\
\hline High, Standard & & & $0.859(0.197)$ & \\
\hline Low, Translational & & & $0.127^{* * * *}(0.055)$ & \\
\hline Medium, Translational & & & $0.210^{* * * *}(0.083)$ & \\
\hline High, Translational & & & $0.207^{* * * *}(0.078)$ & \\
\hline $\begin{array}{l}\text { Orientation } \\
\text { Base: Clinical }\end{array}$ & $1.084(0.162)$ & $1.313^{*}(0.188)$ & $1.316^{*}(0.183)$ & $1.285^{*}(0.180)$ \\
\hline Cumulative & $1.092(0.160)$ & $1.164(0.152)$ & $1.190(0.149)$ & $1.150(0.149)$ \\
\hline Age & $1.026(0.044)$ & $1.028(0.041)$ & $1.038(0.039)$ & $1.028(0.040)$ \\
\hline \multicolumn{5}{|l|}{$\begin{array}{l}\text { Team-Size } \\
\text { Baseline: Small }\end{array}$} \\
\hline Medium & $0.999(0.151)$ & $1.039(0.157)$ & $1.035(0.156)$ & $1.057(0.158)$ \\
\hline Large & $2.730^{* * *}(0.510)$ & $2.283^{* * *}(0.382)$ & $2.314^{* * *}(0.381)$ & $2.298^{* * *}(0.378)$ \\
\hline Years from Publication & (exposure) & (exposure) & (exposure) & (exposure) \\
\hline Lab Leader (dummies) & YES & YES & YES & YES \\
\hline lnalpha & $0.098(0.080)$ & $0.020(0.075)$ & $-0.007(0.074)$ & $-0.006(0.076)$ \\
\hline alpha & $1.103(0.088)$ & $1.020(0.077)$ & $0.993(0.074)$ & $1.006(0.076)$ \\
\hline Log pseudolikelihood & -1447.11 & -1432.07 & -1426.67 & -1429.24 \\
\hline Wald chi2 & $130.75(12)$ & $176.04(18)$ & $194.30(20)$ & $193.77(19)$ \\
\hline
\end{tabular}

Based on calculations on the coefficients in these regression models, we can gauge the effect of the key explanatory variables in each approach as well as the difference between approaches, at every level of 
the key explanatory variables. We test the significance of these estimates with a Wald test. The results are reported in Table 5.

Table 5 Effect of interacted variables in the translational and the standard approach. Results of Wald tests

\begin{tabular}{|c|c|c|}
\hline Model & Relationship & IRR (Std. Err.) \\
\hline & Diversity X Approach & $1.650^{* *}(0.348)$ \\
\hline Model 3 & Medium, Translational vs. Low, Translational & $1.626^{* *}(0.397)$ \\
\hline Model 3 & High, Translational vs. Low, Translational & $0.985(0.184)$ \\
\hline Model 3 & High, Translational vs. Medium, Translational & $1.426(0.365)$ \\
\hline Model 3 & High, Standard vs. Medium, Standard & $0.919(0.166)$ \\
\hline & Multiple (disciplinary area) x Approach & \\
\hline Model 4 & Multiple, Translational vs. Oncology, Translational & \\
\hline
\end{tabular}

Model-1 shows that only the controls for large size of co-author team have a positive and strongly significant impact on citations. All the subsequent models confirm this effect and show also that basicoriented papers are expected to receive about $30 \%$ more citations than those with a clinical orientation.

Model-2 shows that adoption of a translational approach is highly penalizing in terms of scientific impact; all other things being equal, a translational paper receives about one quarter of the citations received by a paper based on standard research. We find also that Disciplinary Area strongly affects the acceptance and diffusion of the work in the scientific community. Compared to contributions specialized in Oncology, papers addressing issues related to Practice-Group have an expected citation rate more than two times higher while articles on Disease-Field and Multiple disciplinary areas are penalized compared to contributions to Oncology.

In Model-3 we examine Hypothesis 1 by comparing the two approaches in terms of the effect of input complementarities (the three levels of Diversity derived from the Diversity Index) on subsequent 
scientific impact. To make this comparison we draw on the Wald tests of differences between the estimates presented in Table 4.

For the translational approach these tests show that studies with medium Diversity are expected to receive $65 \%$ more citations than low Diversity papers; this citation rate is not significantly different from that of high Diversity studies - which are expected to receive $62 \%$ more citations than low Diversity studies. By contrast, in the standard approach, higher diversity does not mean a stronger impact. Indeed, medium and higher levels of diversity show lower impact compared to low level diversity, but this penalizing effect is significant only for the medium level where a $40 \%$ decrease is observed. Hence, confirming Hypothesis 1, exploitation of cognitive complementarities does increase the scientific impact in a new science, whereas a similar effect is not found for standard science.

Hypothesis 2 refers to differences between the two approaches in the relationship between subject scope and scientific impact. To understand whether the penalizing effect of broad scope investigations is specific to one of the approaches, the interaction in Model 4 between Multiple and Approach shows that articles based on the standard approach covering multiple disciplinary areas are expected to receive about 55\% fewer citations than articles specialized in Oncology. The Wald test reported in Table 4 shows that, in translational research there is no penalty associated with explorations outside disciplinary areas. Table 1 showed that the nature of studies with broad Scope differs between the translational and the standard approach, in the former case representing a form of RT-driven research, and in the latter exploration of issues spanning several disease areas and clinical practices. The model indicates that, in the translational approach, both studies focused on Oncology and those covering multiple areas are expected to receive almost $80 \%$ fewer citations than a standard study specialized in Oncology. These results provide support for Hypothesis 2, of a negative effect of broad subject scope in the standard approach compared to 
Translational Medicine which appears to be able to accommodate broad scope subjects in its research agenda. It emerges that translational scientists can move out the core domain of Oncology towards multiple fields without compromising their expected citation impact. Absence of a penalizing effect is associated also with investigations in RT-fields - undertaken only in translational research - while another model (not presented here) indicates that the payoff from investigations in the Disease-Fields is negative for both approaches, but significantly less rewarding for the standard (-60\%) than the translational approach $(-40 \%)$. Overall these results seem to suggest a better ability of Translational Medicine to broaden the scope of investigation.

Finally, we observe that all the models consistently show a penalty related to the citation rate associated with Translational Medicine. This penalty is attached to the translational approach independent of the cognitive configuration of the focal paper in terms of cognitive complementarities or scope. The lower impact of translational studies may be a consequence of the divergence of its selection criteria from those of standard research, combined with the its comparatively smaller scientific constituency. Indeed, a field in its early stages of evolution is not likely to have developed a broad scientific community and so seeks recognition from other related communities.

\section{Discussion and conclusion}

The emergence of Translational Medicine allows us to analyze an aspect of the relationship between science and technology that has received limited interest in Innovation Studies. The history of the Life Sciences in the second half of the $20^{\text {th }}$ century offers an example of the incorporation of fundamental scientific advances into a set of new research technologies. For this reason it provides a window onto the reverse effect of how these research technologies affect the production and reception of scientific 
research. Our study provides evidence of the tight linkage between a new science, such as Translational Medicine, and a set of research technologies.

The research technologies based on advances in Molecular Biology have enabled the exploration of phenomena that cut across established disciplinary domains. Rather than being homogeneous, the adoption of these research technologies has spurred the formation of distinct epistemic communities with specific characterizations in terms of the object of investigation, methodological approach, and impact on subsequent developments in the field.

Research technologies are an important driver of diversity in the knowledge base of a new science, and enable broadly scoped scientific investigations. Translational Medicine extensively relies on technologydriven cognitive complementarities, differently from standard medical research that exploits these complementarities only marginally. Furthermore, we find that the criteria defining scientific advances and their importance in translational medicine are different from those found in "standard" medical research, as only the former relies on cognitive complementarities as a driver of scientific impact. In other words, we find that Translational Medicine and standard medical research are distinct approaches not simply because they apply very different research technologies, but because scientific impact takes quite different cognitive forms in the two approaches. These results provide empirical evidence supporting the conceptualization of Translational Medicine as a "new science" (Bonaccorsi 2010). However, we do not consider other features of the new sciences, namely rate of growth and theoretical proliferation. Further studies aimed at characterizing a field as a "new science" should measure these additional dimensions. 
We found also that new sciences address issues that are relevant for the development of research technologies, as shown by the high share of translational articles contributing to RT-Fields; by contrast, contributions to knowledge on research technologies is not part of the established sciences agenda, which maintains a strong focus on its main disciplinary field, in our case Oncology. An important finding of our work is that broadening the subject matter to issues that span multiple disciplines detracts from the impact of established sciences but does not affect new sciences. This cognitive feature implies that the two approaches represent different regimes of knowledge production. Standard research seems to progress via contributions that deepen knowledge in Oncology and closely connected areas, while translational scientists appear more free to investigate a broader space of disciplinary areas. This result seems to confirm that adoption of research technologies opens the opportunity to investigate complex problems that are at the interface between different disciplines.

The high penalty associated with adoption of a translational approach, all other things being equal, is another important finding of our study. From the perspective of Evolutionary Epistemology, this indicates that the outcomes of translational studies do not conform to the criteria currently employed by Medicine to select relevant contributions. As other new sciences, Translational Medicine has emerged "inside" old sciences in the form of a smaller and institutionally more tenuous community. We suggest that the co-existence of distinct models of knowledge production within the same field of medical research may reduce the opportunities for intellectual exchange and limit the space for knowledge combinations. When selection criteria are as dramatically different as we find in this study, the much larger size of the established old science brings about a stronger overall impact and acceptance of its research. In other words, it becomes apparent that Translational Medicine is still far from achieving intellectual maturity. 
The different potential causes for the weaker impact of translational medicine should be disentangled in further research. Whatever the answers derived, we observe a misalignment between the priorities of science policy and the positions of the most influential scientific opinion leaders, which advocates for increasing diffusion of the translational approach, and the internal rewards offered by the scientific system. So far, policy debate on translational research emphasizes the need to broaden the competence portfolios of scientists through appropriate education so that they can master multiple areas of the Life Sciences. Another issue highlighted by this debate concerns the high complexity of the ultimate goal of medical research, prevention and treatment of disease, so that even an interdisciplinary approach such as translational medicine might not be sufficient to tackle such a complex problem. Our study highlights another constraint on the diffusion of the translational approach, i.e. the disincentives offered to scholars by the academic community. This finding provides an additional rationale for policy support for Translational Medicine. Further research on this topic is needed to appreciate the effectiveness of these instruments and to identify the individual characteristics of scientists who decide to adopt the emerging approach. Indeed, deeper knowledge of the resource endowments and motivations of scientists embracing an emerging approach - compared to those pursuing the standard approach - would help our understanding of the patterns of diffusion of new research technologies. The literature on these issues focuses more on the consequences of than the antecedents to the adoption of research technologies.

Comparing the scientific impact of the various cognitive drivers, we see that in both approaches scientists pursue research strategies which do not fully realize the potential scientific impact from their comparative advantage. We showed that Scope has a neutral effect on impact in Translational Medicine and a negative effect in standard clinical research; but the two approaches have produced a very similar share of papers (about one-fifth) spanning multiple fields. Moreover, we find that the majority of translational 
publications presents a high level of Diversity, despite the fact that a medium level of Diversity is more rewarding and implies lower cognitive costs. In standard studies, the rewards associated with low and high Diversity are similar, while a medium level is penalizing. However, medium Diversity accounts for more papers than high Diversity. Overall, these results suggest that the full potential of scientific contributions associated with their particular comparative advantages may not be being realized by scientists operating in a changing field such as Medicine. There seems to be an absence of "research strategizing" in the sense that scientists in neither approach capitalize efficiently on their comparative advantages.

We showed that there are systematic penalties associated with Translational Medicine compared to the standard approach. So why do translational and clinical studies at the descriptive level (Table 1) present similar average performance? Our regressions show that orientation towards more basic issues is an important driver of impact, and by definition, the production of the two approaches differs significantly along this dimension. The lack of significant differences along other drivers of impact suggests that an important source of variability in performance lies in the characteristics of Lab Leaders and their teams. In this study we considered some of these organizational features, compatible with the restrictions of a quantitative approach: we included controls for size of teams and for team leader. Further research could investigate these dynamics in more depth by employing of qualitative studies focused, e.g. on the mechanisms deployed by teams to effectively integrate different sources of knowledge.

The discrepancy between the descriptive statistics and the results of the econometric analysis is a strong reminder to be cautious about citations data in research evaluations. These exercises should carefully consider possible confounding factors affecting performance and use appropriate statistical tools. 
Finally, we should emphasize the limited generalizability of our results. Our empirical analysis is based on the production of a small sample of Lab Leaders. The selection of cases capitalizes on the possibility to refer Lab Leaders precisely to either one or the other approach. The drawback of this methodology is the limitations it imposes on generalizability to Medicine. Large-scale studies are needed to validate our findings. The elaboration of objective indicators capable of capturing the "translational" nature of a piece of research would seem a necessary initial step in such a research program. 


\section{Appendix-I: Procedures for selecting Laboratory-Leaders}

We defined the subfield of Breast Cancer Research searching for journal articles indexed in ISI-WoS with the words "breast cancer" or synonyms in their title or abstract, published in 2003-2007. We generated a list of the most prolific European Authors; We then identified those who published at least two-thirds of their production in Breast Cancer Research and could thus be considered "specialized". We considered only European cases to avoid influence from the notable differences between Europe and the USA regarding the organization and funding of medical research (Owen-Smith et al. 2002). To avoid country-specific effects, we did not allow the same country to be represented in the translational and the standard group more than once. Combining publications data and other sources of documentation - such as institutional and personal websites, and affiliation to medical societies - we identified Laboratory Leaders, i.e. scientists who establish a stable team of collaborators endowed with technological and financial resources and lend to the group a shared vision and a coherent strategy, unifying the contributions of specialists. We excluded from the definition scientists who had co-authored a substantial share of their production with more prolific scientists, or those responsible of larger organizations, such as entire departments.

We identified Laboratory Leaders who represented each approach on the basis of the predominant CHILevel of their production. Translational Laboratory Leaders are required to publish at least $25 \%$ of their work in Levels-3 or 4. Furthermore, the principles and organization of their work must be translational, as per publicly available documents on their research units. "Standard" Laboratory Leaders were identified among those with fewer than $25 \%$ of their articles in CHI-3 and 4 and indicating no engagement in translational objectives or organization of their work. 
The set of translational contributions then is a blend of CHI- 2 and 3 articles with a marginal presence of clinical observations and basic research. The control set concentrates $75 \%$ of its articles in CHI-2, and the remainder is split evenly between clinical observations and clinical investigations, with no basic research. This comparison suggests a more pronounced attitude of translational Laboratory Leaders to span different CHI-Levels, and higher intensity of analytical-oriented studies.

We identified three translational Laboratory Leaders, for whom we collected complete publication records from 2003 to 2007. Given the lower productivity of "standard" scientists meeting the selection criteria, we considered five cases in order to gather a comparable set of publications. We checked for homonymy and we included three publications not appearing in ISI-WoS records because of misspelt names.

We obtained 184 translational and 200 "standard" articles - a total of 384 . We excluded articles with more than 50 co-authors because they can hardly be considered the result of real collaboration: translational Laboratory Leaders had 4 such articles and the other group 12 . We finally obtained a valid dataset of 356 articles. 


\section{Appendix-II: Attribution of Subject-Categories to Disciplinary-Specializations}

\begin{tabular}{|l|l|}
\hline Oncology & \\
\hline Oncology & Biotechnology \& Applied-Microbiology \\
\hline Disease-Fields & Cell \& Tissue Engineering \\
\hline & Cell Biology \\
\hline Endocrinology \& Metabolism & Genetics \& Heredity \\
\hline Gastroenterology \& Hepatology & Medical Laboratory Technology \\
\hline Hematology & Microbiology \\
\hline Immunology & Reproductive Biology \\
\hline Obstetrics \& Gynecology & \\
\hline Peripheral Vascular Disease & Practice-Group \\
\hline Psychology & \\
\hline Psychology, Multidisciplinary & Health Care Sciences \& Services \\
\hline Public, Environmental \& Occupational Health & Medicine, General \& Internal \\
\hline Respiratory System & Medicine, Research \& Experimental \\
\hline & Pathology \\
\hline RT-Fields & Pharmacology \& Pharmacy \\
\hline & Physiology \\
\hline Biochemical Research Methods & Radiology, Nuclear Medicine \& Medical Imaging \\
\hline Biochemistry \& Molecular Biology & Rehabilitation \\
\hline Biophysics & Surgery \\
\hline
\end{tabular}




\section{References}

Adams JD, Black GC, Clemmons JR and Stephan PE. (2005). Scientific teams and institutional collaborations: Evidence from U.S. universities, 1981-1999. Research Policy, 34(3): 259-285.

Aksnes DW and Rip A (2009). Researchers' perceptions of citations. Research Policy, 38(6): 895-905.

Amir-Aslani A and Mangematin V (2010). The future of drug discovery and development: Shifting emphasis towards personalized medicine. Technological Forecasting and Social Change 77(2):203-217.

Bonaccorsi A (2008). Search Regimes and the Industrial Dynamics of Science. Minerva, 46(3):285-314.

Bonaccorsi A (2010). New Forms of Complementarity in Science. Minerva, 48:355-387.

Bonaccorsi A and Thoma G (2007). Institutional complementarity and inventive performance in nano science and technology. Research Policy, 36:813-831.

Bonaccorsi A and Vargas J (2010). Proliferation dynamics in new sciences. Research Policy, 39(8):10341050

Boyack KW (2005). Mapping the backbone of science. Scientometrics 64(3):351-374.

Bradie M (1986). Assessing evolutionary espistemology. Biology and Philosophy, 1(4): 401-459.

Callon M (1994). Is Science a Public Good? Fifth Mullins Lecture, Virginia Polytechnic Institute, 23 March 1993. Science, Technology \& Human Values, 19(4): 395-424.

Campbell DT (1974). Evolutionary Epistemology. In Schlipp PA (ed.) The philosophy of Karl R. Popper. Open Court, La Salle (IL), pp 412-463. 
Cambrosio A, Keating P, Mercier S, Lewison G and Mogoutov A (2006). Mapping the emergence and development of translational cancer research. European Journal of Cancer, 42: 3140-3148.

Coccia, M (2014). Converging scientific fields and new technological paradigms as main drivers of the division of scientific labour in drug discovery process: the effects on strategic management of the R\&D corporate change. Technology Analysis \& Strategic Management, 26(7): 733-749.

Coccia M, and Wang L (2014). Path-breaking directions of nanotechnology-based chemotherapy and molecular cancer therapy. Technological Forecasting and Social Change. 94: 155-169.

Collins R (1994). Why the social sciences won't become high-consensus, rapid-discovery science. Sociological Forum 9(2):155-177.

Consoli D and Ramlogan R (2008). Out of sight: problem sequences and epistemic boundaries of medical know-how on glaucoma. Journal of Evolutionary Economics, 18(1): 31-56.

Consoli D and Ramlogan R (2012). Patterns of organization in the development of medical know-how: the case of glaucoma research. Industrial and Corporate Change, 21(2): 315-343.

Dasgupta P and David PA (1994). Toward a new economics of science. Research Policy, 23: 487-521.

De Solla Price DJ (1963). Little Science, Big Science. Columbia University Press, New York.

Douglas CMW, Lander B, Fairley C and Atkinson-Grosjean J (2014). The Roles of User/Producer Hybrids in the Production of Translational Science. Social Epistemology. DOI: $10.1080 / 02691728.2013 .848951$. 
Faulkner A (2009). Regulatory policy as innovation: Constructing rules of engagement for a technological zone of tissue engineering in the European Union. Research policy, 38(4): 637-646.

Faulkner A (2015). Usership of regenerative therapies: Age, ageing and anti-ageing in the global science and technology of knee cartilage repair. Technological Forecasting and Social Change, 93: 44-53.

Funtowicz SO and Ravetz JR (1993). Science for the post-normal age. Futures, 25(3):739-755.

Gelijns A and Rosenberg N (1999). Diagnostic devices: an analysis of comparative advantages. In Mowery D and Nelson RR. The Sources of Industrial Leadership. Cambridge University Press. 312-358.

Gittelman M and Kogut B (2003). Does good science lead to valuable knowledge? Biotechnology firms and the evolutionary logic of citation patterns. Management Science, 49(4):366-384.

Grimes K (2011). UK's biomedical research institute gets green light. The Lancet, 377(9762):288.

Heimeriks G (2012). Interdisciplinarity in biotechnology, genomics and nanotechnology. Science and Public Policy, 39(4):1-16.

Heimeriks G and Leydesdorff L (2012). Emerging search regimes: measuring co-evolutions among research, science, and society. Technology Analysis and Strategic Management, 24:51-67.

Henderson R, Orsenigo L and Pisano GP (1999). The Pharmaceutical Industry and the Revolution in Molecular Biology: Interactions among Scientific, Institutional and Organizational Change. In: Mowery DC and Nelson RR (Eds.) Sources of Industrial Leadership. Cambridge University Press, Cambridge UK, pp. 267-311. 
Hopkins MM, Martin PA, Nightingale P, Kraft A, Mahdi S (2007). The myth of the biotech revolution: An assessment of technological, clinical and organisational change. Research Policy 36(4):566-589.

Horlings E and Gurney T (2013). Search strategies along the academic lifecycle. Scientometrics 94(3):1137-1160

Jansen D, von Gortz R and Heidler R (2010). Knowledge production and the structure of collaboration networks in two scientific fields. Scientometrics, 83: 219-241.

Joerges B and Shinn T (2001). Instrumentation between science, state and industry. Kluwer Academic Publishers Group, Dordrecht.

Jones DS, Cambrosio A and Mogoutov A (2011). Detection and characterization of translational research in cancer and cardiovascular medicine. Journal of Translational Medicine, 9: 57.

Judson HF (1979). The eighth day of creation. Makers of the revolution in biology. Penguin Books, London.

Klein J (2008). Evaluation of Interdisciplinary and Transdisciplinary Research: A Literature Review. American Journal of Preventive Medicine, 35(2):116-123.

Kukk P, Moors EHM and Hekkert MP (2015). The complexities in system building strategies-The case of personalized cancer medicines in England. Technological Forecasting and Social Change, 98: 47-59.

Lakatos I and Musgrave A (1970). Criticism and the growth of knowledge: proceedings of the International Colloquium in the Philosophy of Science, London, 1965. Vol. 4. Cambridge University Press, Cambridge. 
Larivière V and Gingras Y (2010). On the Relationship Between Interdisciplinarity and Scientific Impact. Journal of the American Society for Information Science and Technology, 61(1):126-131.

Lehoux P, Daudelin G, Williams-Jones B, Denis JL and Longo C. (2014). How do business model and health technology design influence each other? Insights from a longitudinal case study of three academic spin-offs. Research Policy, 43(6): 1025-1038.

Lepori B (2011). Coordination modes in public funding systems. Research Policy 40(3):355-367.

Levitt J and Thelwall M (2008). Is multidisciplinary research more highly cited? A macrolevel study. Journal of the American Society for Information Science and Technology, 59(12):1973-1984.

Lewison G, Purushotham A, Mason M, McVie G and Sullivan R (2010). Understanding the impact of public policy on cancer research: A bibliometric approach. European Journal of Cancer, 46(5): 912-919.

Llerena P and Meyer-Krahmer F (2003). Interdisciplinary research and the organizationof the university: General challenges and a case study. In: Geuna A, Salter AJ and Steinmueller WE (eds.), Science and Innovation. Rethinking the Rationales for Funding and Governance. Edward Elgar, Cheltenham.

Long JS and Freese J (2006). Regression models for categorical dependent variables using Stata. Stata Press, College Station (TX).

Luwel M and van Wijk E (2015). The 'translational' effect in medical journals: Bridging the gap? Research Evaluation 24(1): 19-29.

Marincola F (2007). In support of descriptive studies; relevance to translational research. Journal of Translational Medicine, 5(1): 21. 
McLeod HL, Tortora G, Rowett LH and Vermorken JB (2011). Found in translation: Annals of Oncology and translational research. Annals of Oncology, 22(8): 1698-1699.

Merton RK (1957). Priorities in scientific discovery: a chapter in the sociology of science. American Sociological Review, 22(6): 635-659.

Metcalfe JS, Andrew J and Mina A. (2005). Emergent innovation and system and the delivery of clinical services: The case of intra-ocular lenses. Research Policy, 34(9): 1283-1304.

Meyer M (2007). What do we know about innovation in nanotechnology. Some propositions about an emerging field between hype and path-dependency. Scientometrics, 70(3): 779-810.

Moran N (2007). Public sector seeks to bridge 'Valley of death'. Nature Biotechnology, 25(3): 266.

Morange M (1998). A History of Molecular Biology. Harvard University Press, Cambridge (MA).

Morlacchi P and Nelson RR (2011). How medical practice evolves: Learning to treat failing hearts with an implantable device. Research Policy, 40(4): 511-525.

Nahuis R and Stemerding D (2013). Genomics as a new research regime? Evidence from the Netherlands. Research policy, 42(3): 676-687.

Nelson RR, Buterbaugh K, Perl M and Gelijns A (2011). How medical know-how progresses. Research Policy 40(11):1339-1344.

Nicolini D (2010). Medical innovation as a process of translation: A case from the field of telemedicine. British Journal of Management, 21(4): 1011-1026. 
Peine A, Faulkner A, Jæger B, and Moors E. (2015). Science, technology and the 'grand challenge'of ageing-Understanding the socio-material constitution of later life. Technological Forecasting and Social Change, 93: 1-9.

Ponomariov BL and Boardman PC (2010). Influencing scientists' collaboration and productivity patterns through new institutions: University research centers and scientific and technical human capital. Research Policy, 39(5):613-624.

Porter AL, Rafols I (2009). Is science becoming more interdisciplinary? Measuring and mapping six research fields over time. Scientometrics 81(3):719-745.

Pudovkin AI, Garfield E (2002). Algorithmic procedure for finding semantically related journals. Journal of the American Society for Information Science and Technology 53:1113-1119.

Rafols I, Hopkins MM, Hoekman J, Sipel J, O’Hare A, Perianes-Rodriguez A and Nightingale P (2014). Big Pharma, little science? A bibliometric perspective on Big Pharma's R\&D decline. Technological Forecasting \& Social Change 81: 22-38.

Ryan MP (2010). Patent incentives, technology markets, and public-private bio-medical innovation networks in Brazil. World Development, 38(8): 1082-1093.

Rosenberg N (1992). Scientific Instrumentation and University Research. Research Policy 21: 381-390.

Sabatier V, Craig-Kennard A and Mangematin V (2012). When technological discontinuities and disruptive business models challenge dominant industry logics: Insights from the drugs industry. Technological Forecasting and Social Change, 79(5): 949-962. 
Sablinski T (2014). Opening Up Clinical Study Design to the Long Tail. Science Translational Medicine, 6(256): 256.

Schilling MA, Vidal P, Ployhart RE and Marangoni A (2003). Learning by doing something else: variation, relatedness and the learning curve. Management Science 49(1):39-56.

Schoenmakers W and Duysters G. (2010). The technological origins of radical inventions. Research Policy, 39(8): 1051-1059.

Shinn T (2005). New Sources of Radical Innovation: Research Technologies, Transversality and Distributed Learning in a Post-Industrial Order. Social Science Information, 44(4):731-734.

Sternitzke C (2010). Knowledge sources, patent protection, and commercialization of pharmaceutical innovations. Research Policy, 39(6):810-821.

Stokes DE (1997). Pasteur's Quadrant. Basic Science and Technological Innovation. The Brookings Institution, Washington D.C.

Thagard PR (1999). How scientists explain disease. Princeton University Press, Princeton.

Tierney R, Hermina W and Walsh S (2013). The pharmaceutical technology landscape: A new form of technology roadmapping. Technological Forecasting and Social Change, 80(2):194-211.

Tijssen RJW (2010). Discarding the 'basic science/applied science' dichotomy: A knowledge utilization triangle classification system of research journals. Journal of the American Society for Information Science and Technology, 61(9):1842-1852. 
van der Valk T, Chappin MMH and Gijsbergs GW (2011). Evaluating innovation networks in emerging technologies. Technological Forecasting \& Social Change, 78(1):25-39.

Webb C and Pass H (2004). Translation research: from accurate diagnosis to appropriate treatment. Journal of Translational Medicine, 2(1):35.

Whitley R (2000). The Intellectual and Social Organisation of the Sciences. Oxford University Press, Clarendon. 